\title{
CONTROLE PREDITIVO VIA PROGRAMAÇÃO MULTIPARAMÉTRICA APLICADO NO MODELO CINEMÁTICO DE UMA CADEIRA DE RODAS ROBÓTICA
}

\author{
GUSTAVO P. C. WEINKELLER ${ }^{1}$, JOSÉ LEANDRO F. SALLES ${ }^{1}$, TEODIANO F. B. FILHO ${ }^{1}$
}

\author{
1. Programa de Pós-Graduação em Engenharia Elétrica, UFES - Universidade Federal do Espirito \\ Santo \\ Av. Fernando Ferrari, 514, CEP 29075-910. Vitória - ES \\ E-mails: gustavocola@gmail.com,jleandro/tfbastos@ele.ufes.br
}

\begin{abstract}
This paper proposes the use of an explicit kinematic predictive control, which is a PWA (PieceWise Affine) function of the dynamic model states of a robotic wheelchair. The optimization algorithmic is based on Multi-parametrical Programming, allowing all calculations of this controller be performed off-line, before starting the system operation, resulting in a reduction of online computational time. Some simulation and experimental results illustrate the effectiveness of the proposed method
\end{abstract}

Keywords— Robot Wheelchair, Predictive Control, PieceWise Affine systems, Multi-parametric Programming

Resumo - Normalmente a implementação de um controlador preditivo em sistemas com dinâmica rápida é inviável devido à quantidade de cálculos necessários para se determinar a solução do problema de controle. Este artigo mostra um controlador preditivo dinâmico explícito, do tipo PWA (PieceWaise Affine), atuando no modelo cinemático de uma cadeira de rodas robotizada O algoritmo de otimização utilizado foi a Programação Multiparamétrica, permitindo assim que todos os cálculos deste controlador fossem realizados explicitamente ou off-line, antes do início do sistema, resultando assim em economia de tempo computacional online.

Palavras-chave—Cadeira de rodas robótica, Controle preditivo, sistemas afins por partes, programação multiparamétrica

\section{Introdução}

Diversos segmentos da ciência estudam uma melhor forma de viabilizar a inserção de pessoas com limitações físicas na sociedade nas quais estão inseridas, facilitando sua comunicação, locomoção e sua interação social como um todo. Uma dessas vertentes tem seu foco voltado para cadeiras de rodas inteligentes. Essas cadeiras inteligentes podem se locomover sozinhas ou sob comandos específicos, como por exemplo, comandos dados por posicionamento ocular, respiração, ondas cerebrais, dentre outros.

Um robô móvel do tipo cadeira de rodas é composto por duas rodas motrizes de tração diferencial atrás e duas rodas direcionais livres na frente. Esse tipo de robô é de fácil movimentação e a configuração das rodas se dá de forma simples. Apesar das suas vantagens, este tipo de robô apresenta um sistema não linear (CELESTE, 2009) e (MARTINS, et al., 2008).

No caso de uma cadeira de rodas robótica, manter a estabilidade e ter um sistema que responda rápido a perturbações externas é fundamental, tendo em vista que seu ocupante possa se movimentar, que pessoas possam esbarrar na mesma, dentre outros. Questões de segurança e conforto também têm de ser levadas em consideração, e para isso sua velocidade linear e velocidade angular são restringidas em $v=[-0.4 \mathrm{~m} /$ $s, 0.4 \mathrm{~m} / \mathrm{s}]$ e $\omega=[-0.5 \mathrm{rad} / \mathrm{s}, 0.5 \mathrm{rad} / \mathrm{s}] \mathrm{respecti-}$ vamente. (CELESTE, 2009) e (MARTINS, et al., 2008)

O controlador preditivo é utilizado com sucesso em sistemas não lineares restritos, sob efeito de perturbações e com múltiplas entradas e múltiplas saídas.
Entretanto, sua utilização em sistemas de dinâmica rápida pode ser um problema devido à utilização da Programação Quadrática como forma de otimização. Neste caso, o valor do Horizonte de previsão $\left(h_{p}\right)$, do Horizonte de controle $\left(h_{c}\right)$ e a quantidade de entradas, saídas e restrições, influenciam diretamente na quantidade de cálculos online necessários para resolver a solução do problema de controle. Tal fato pode inviabilizar a utilização do controlador preditivo por limitações de hardware e pela demora na determinação de um sinal de controle. (CAMACHO \& BORDONS, 2004)

Contudo, devido aos avanços computacionais e a novos algoritmos de otimização, o controle preditivo vem sendo empregado com bons resultados em robótica móvel. Alguns deles tratam especificamente da cinemática de um robô móvel, seja seguindo um valor de referência (KÜHNE, et al., 2005) e (Akiba, et al., 2010) ou uma trajetória específica (KLANCAR \& SKRJANC, 2007), (Rico, et al., 1999) e (KANJANAWANISHKUL \& ZELL, 2008).

Outras ferramentas vêm sendo empregadas em conjunto com o controlador preditivo, como é o caso de redes neurais, lógica Fuzzy, algoritmo genético, inteligência artificial, dentre outros. Como a modelagem de robôs móveis normalmente resultam em complexas equações diferenciais, estas ferramenta se mostram atrativas na identificação e levantamento dos parâmetros de tais sistemas. Outra aplicação interessante para essas ferramentas é a determinação dos parâmetros ótimos do controlador, como seus pesos e horizontes. (CHEN \& LI, 2007) e (YOO, et al., 2006)

Uma interessante aplicação de controle preditivo hibrido foi implementada em futebol de robôs, sendo responsável pelo controle da postura e da velocidade 
dos robôs. Esse controlador fui subdividido em três partes; o Generalized Predictive Control (GPC), responsável pelo controle da trajetória; o Support Vector Machine, responsável pela determinação do ângulo de posição ótimo; e o Fuzzy Logic Controller, responsável pelo controle da velocidade do robô, obtendo bons resultados. (CHIOU \& WANG, 2008)

Neste artigo será mostrada uma abordagem de controlador preditivo baseado no modelo de espaço de estados discreto do sistema cinemático de uma cadeira de rodas, onde o método de otimização é conhecido por Programação Multiparamétrica (PM), mais indicado para sistemas com dinâmica rápida.

A PM tem como objetivo determinar uma solução ótima para um problema de controle de forma explícita. Assim, se a representação da solução ótima é obtida explicitamente, e conhecendo-se a equação de estados do sistema, o cálculo da ação de controle se reduz em avaliar qual é ação de controle em cada estado onde se encontra o sistema. Desta forma o algoritmo de PM encontrará uma solução mais rapidamente do que outros algoritmos. (KVASNICA, 2009)

O controlador obtido usando PM é dinâmico e denominado PieceWaise Affine (PWA). A ação de controle previamente determinada é uma função dos estados $x$ do sistema. Os estados factíveis compõem um politopo que é separado em setores politópicos, cada qual possuindo uma ação de controle específica. Logo, a quantidade de cálculos necessários para se estipular uma ação de controle de um determinado estado do sistema está relacionado com a quantidade de setores politópicos. A quantidade de setores politópicos está diretamente relacionada com o $h_{p}, h_{c}$, e a quantidade de entradas, saídas e restrições. (SICILIANO \& KHATIB, 2008)

\section{Formulação do problema}

Para se entender o movimento de um robô, deve-se entender a contribuição de cada roda no movimento, pois são elas as responsáveis pelo deslocamento do robô como um todo. Da mesma forma que a roda é responsável por gerar o movimento, ela também gera restrições como, por exemplo, o fator de escorregamento lateral e as próprias limitações impostas pelo seu tipo e tamanho (NOURBAKHSH e SIEGWART, 2004).

Partindo do princípio que o robô é um corpo rígido sobre rodas não deformáveis e que a superfície na qual está inserido é um plano horizontal, assumese que a postura ${ }^{1}$ do robô tem três dimensões, duas coordenadas que representam sua posição no referido plano e um ângulo que determina sua orientação. Sendo assim, pode-se representar a postura de um robô móvel pelo vetor

$$
\xi=\left[\begin{array}{l}
x \\
y \\
\psi
\end{array}\right]
$$

onde $x$ representa a posição ao longo do eixo das abicissas, $y$ representa a posição ao longo do eixo das ordenadas e $\psi$ seu ângulo de orientação em relação ao eixo das abcissas.

No caso de uma cadeira de rodas robótica, a cinemática de seu comportamento é peculiar, isso se dá pelo fato de haver uma carga pesada e móvel, uma pessoa, que pode vir a representar até $60 \%$ da massa do sistema cadeira de rodas robótica mais usuário. Devido a esse fato, é fundamental levar em consideração a presença do usuário no sistema dinâmico do robô. A Figura 1 mostra o esquema da cadeira de rodas (CELESTE, 2009).

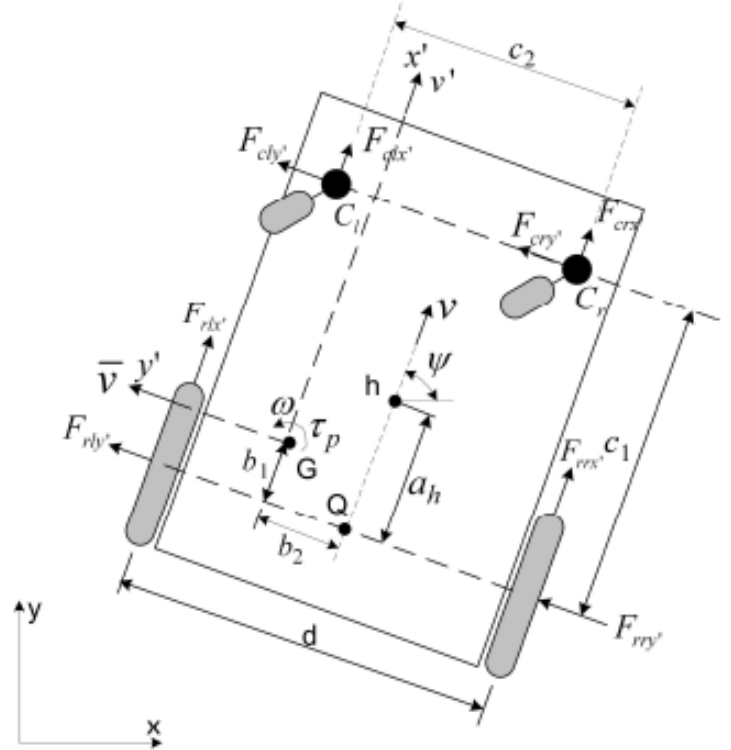

Figura 1. Parâmetros de uma cadeira de rodas robótica. (CELESTE, 2009)

A partir das informações da figura 1, o modelo cinemático da cadeira de rodas robótica é obtido (CELESTE, 2009),

$$
\left\{\begin{array}{l}
\dot{x}=v \cos \psi-a_{h} \omega \operatorname{sen} \psi+\delta_{x} \\
\dot{y}=v \operatorname{sen} \psi+a_{h} \omega \cos \psi+\delta_{y}, \\
\dot{\psi}=\omega
\end{array}\right.
$$

sendo $\delta_{x}=-\bar{v}^{s} \operatorname{sen} \psi, \delta_{y}=\bar{v}^{s} \cos \psi$ componentes do distúrbio ocasionado pelo deslizamento lateral das rodas de tração, onde $\bar{v}^{s}$ é a velocidade de deslizamento lateral de tais rodas.

\footnotetext{
${ }^{1}$ Postura: Nome dado ao conjunto das informações das coordenadas no qual está situado um robô e do ângulo que o mesmo faz com um eixo referencial.
} 


\section{Aproximação Linear}

O modelo cinemático descrito por (2) possui não linearidades que devem ser tratadas antes de se transformar o sistema para um modelo em espaço de estados. Para tal é utilizada uma aproximação por série de Taylor em torno dos pontos $(\bar{\varphi}, \bar{v})$. Considerando que as perturbações externas são nulas e descartando os termos de maior ordem da série, obtém-se a expressão linearizada do modelo dinâmico da cadeira de rodas robótica dada por:

$$
\begin{aligned}
\dot{x}= & f_{1}(\bar{\varphi}, \bar{v})-\bar{v} \operatorname{sen} \bar{\varphi}(\varphi-\bar{\varphi})+ \\
& \cos \bar{\varphi}(v-\bar{v}) \\
\dot{y}= & f_{2}(\bar{\varphi}, \bar{v})+\bar{v} \cos \bar{\varphi}(\varphi-\bar{\varphi})+ \\
& \operatorname{sen} \bar{\varphi}(v-\bar{v}) \\
\dot{\varphi}= & \omega
\end{aligned}
$$

Para realizar a linearização, se fez necessário 3 pontos de linearização para $v, \bar{v}_{1}=0, \bar{v}_{2}=0,25 \mathrm{e}$ $\bar{v}_{3}=-0,25$, pontos médios dos intervalos, $I v_{1}=$ $[-0,1,0,1], \quad I v_{2}=[0,1,0,4]$ e $I v_{3}=[-0,4$, $-0,1]$, e 16 intervalos para o ângulo de orientação, totalizando assim 48 intervalos diferentes.

Para o ângulo de orientação tem-se os intervalos

$$
\mathrm{I} \varphi_{i+1}=[22,5 i, 22,5(i+1)]
$$

sendo $i=0,1, \cdots, 15$,

com pontos de operação como sendo os pontos médios de seus intervalos:

$$
\bar{\varphi}_{i+1}=22,5 i+11,25 \text {, }
$$

A Figura 2 mostra um gráfico da simulação do sistema cinemático real comparado ao sistema linearizado em 48 partições distintas e com erros de aproximadamente $1,5 \%$ no deslocamento em horizontal e de $0,8 \%$ no deslocamento vertical.

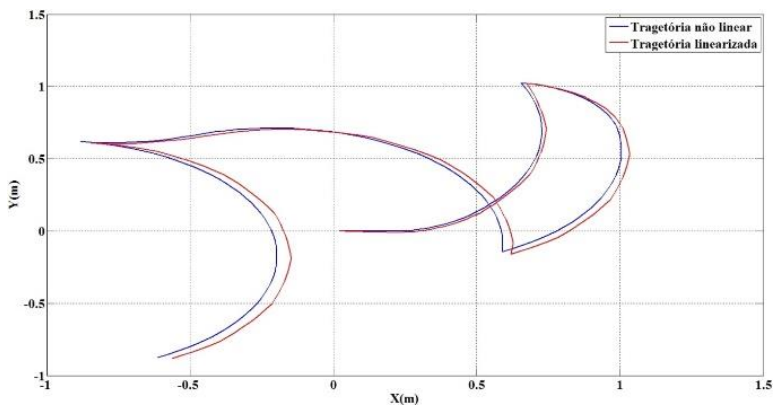

Figura 2. Simulação dos modelos cinemático real e linearizada com 48 partições.

\section{O Problema de Controle Preditivo}

Considere o sistema dinâmico discreto no tempo representado pela equação de estados:

$$
\begin{array}{ll} 
& x_{k+1}=A x_{k}+B u_{k} \\
& y_{k}=C x_{k}+D u_{k} \\
\text { s.a. } & u_{\min } \leq u_{t} \leq u_{\max } \\
& y_{\min } \leq y_{t} \leq y_{\max }
\end{array}
$$

Assuma que este sistema seja controlável e observável, então o problema de Controle Ótimo no Tempo Finito com Restrições (COTFR) é definido através da seguinte formulação:

$$
\begin{aligned}
& J_{h_{p}}^{*}\left(x_{t}, U\right)=\min _{U}\left\{x_{t+h_{p} \mid t}^{\prime} P x_{t+h_{p} \mid t}+\right. \\
& \sum_{k=0}^{h_{p}-1}\left\|Q_{x} x_{t+k \mid t}\right\|_{2}+\left\|Q_{u} u_{t+k \mid t}\right\| \\
& \text { s.a. } \\
& u_{\min } \leq u_{t+k \mid t} \leq u_{\max }, \forall k=0, \ldots, h_{c} \\
& y_{\min } \leq y_{t+k \mid t} \leq y_{\max }, \forall k=0, \ldots, h_{p} \\
& x_{t+k+1 \mid t}=A x_{t+k \mid t}+B u_{t+k \mid t} \\
& y_{t+k \mid t}=C x_{t+k \mid t} \\
& u_{t+k \mid t}=\mathrm{K} x_{t+k \mid t}, h_{c} \leq k \leq h_{p}
\end{aligned}
$$

Onde $h_{p}, h_{c}, h_{r}$ representam os horizontes de previsão, controle e restrição respectivamente, e satisfazem $\quad h_{p} \geq h_{c}$, e $\quad h_{p}-1 \geq h_{r} ; \quad U=$ $\left(u_{t \mid t}, \ldots, u_{t+h_{c} \mid t}\right)^{T}$ é a sequência de controle ótimo a ser determinada, $\mathrm{P}, Q_{x}, Q_{u}$ são matrizes positiva definidas, e K representa um ganho estabilizante de realimentação de estado. O problema (7) é resolvido em cada instante de tempo t, a partir do vetor de estado previsto $x_{t+k}, k=1 \cdots h_{p}$, e a correspondente ação de controle ótima é obtida $U^{*}=\left(u_{t \mid t}^{*}, \ldots, u_{t+h_{c} \mid t}^{*}\right)^{T}$. O sinal aplicado no processo é a primeira ação de controle $u_{t}=u_{t \mid t}^{*}$.

O Ganho de realimentação K e a matriz custo terminal $\mathrm{P}$ são usados para garantir estabilidade para o problema de controle preditivo, sendo obtidos através da solução do problema linear quadrático com horizonte infinito. (BEMPORAD, et al., 2002)

Assim, definindo as matrizes

$$
\tilde{A}=\left[\begin{array}{l}
I \\
A \\
\vdots \\
A^{N}
\end{array}\right], \widetilde{B}=\left[\begin{array}{llll}
0 & \ldots & \ldots & 0 \\
B & 0 & \ldots & 0 \\
\vdots & \vdots & \vdots & \vdots \\
A B & B & \ldots & 0 \\
\vdots & \vdots & \vdots & \vdots \\
A^{N-1} B & A^{N-2} B & \ldots & 0
\end{array}\right]
$$

o problema (7) pode ser escrito como:

$$
\begin{aligned}
& J^{*}\left(x_{t}\right)=x_{t}^{T} Y x_{t}+\min _{U}\left\{U^{T} H U+x_{t}^{T} F U\right\} \\
& \text { s. } a . \\
& G U \leq W+E x_{t},
\end{aligned}
$$


$H=\tilde{B}^{T} \tilde{Q} \tilde{B}+\tilde{Q}_{u}, F=\tilde{A}^{T} \tilde{Q} \tilde{B}$

$Y=\tilde{A}^{T} \tilde{Q} \tilde{A}$

$\tilde{Q}=\operatorname{diag}\left(Q_{x}, \ldots, Q_{x} Q_{N}\right), \tilde{Q}_{u}=\operatorname{diag}\left(Q_{u}, \ldots, Q_{u}\right)$

Seja o conjunto de parâmetros $\mathrm{X}=\left\{x_{t} \in\right.$ $\left.\mathbb{R}^{n} \mid G U \leq W+E x_{t},\right\}$. Considerando $H$ semi-definida positiva, a solução será dada através do seguinte teorema:

Teorema 1, (BEMPORAD, et al., 2002) e (BORRELLI, 2003): Considere o Problema de Programação Multiparamétrica Quadrática (9). Então:

1 - O conjunto de parâmetros factíveis X é um poliedro convexo;

2 - $\mathrm{O}$ conjunto $\mathrm{X}$ é particionado em $\mathrm{R}$ regiões poliedrais, i.e.:

$$
P_{r}=\left\{x \in \mathbb{R}^{n} \mid H_{r} x \leq K_{r}\right\}, r=1, \ldots, R
$$

3 - A solução ótima $U^{*}: \mathrm{X} \rightarrow \mathbb{R}^{p}$ é uma Função Contínua e Afim por Partes (PWA) em relação ao parâmetro x, i.e.

$$
U^{*}(x)=L_{r} x+C_{r}, \quad \text { se } x \in \mathrm{P}_{r}
$$

4- A função custo ótima $J^{*}: X \rightarrow \mathbb{R} e ́$ contínua, convexa e quadrática por partes, i.e.

$$
J^{*}(x)=x^{T} Q_{r} x+L_{r} x+C_{r}, \quad \text { se } x \in \mathrm{P}_{r}
$$

\section{Resultados}

O projeto do controlador foi feito levando-se em consideração o modelo discreto do sistema linear (3) e as simulações foram realizadas usando-se o modelo não linearizado (2).

Para se desenvolver o controlador preditivo para o modelo cinemático da cadeira de rodas, primeiramente estipulou-se quais são as restrições envolvidas no sistema. Por questões de segurança e conforto para o passageiro, a velocidade linear e a velocidade angular têm de ser limitadas entre:

$$
\left\{\begin{array}{l}
-0.4 \mathrm{~m} / \mathrm{s} \leq v \leq 0.4 \mathrm{~m} / \mathrm{s} \\
-0.5 \mathrm{rad} / \mathrm{s} \leq \omega \leq 0.5 \mathrm{rads} / \mathrm{s}
\end{array} .\right.
$$

A escolha do $h_{p}$, e dos pesos para sintonia do controlador foram obtidos por tentativa e erro, tomandose como base a minimização do tempo gasto na realização dos cálculos do controlador (off-line), do tempo gasto por iteração na ação de controle (online) e do erro percentual total entre a referência e a saída do sistema.

Os parâmetros adotados para o controlador foram, a norma, como sendo a infinita, o controle, como sendo do tipo servo ou seguidor, o método de solução, como sendo a solução ótima da função custo para um horizonte finito, $h_{p}=3$ e os pesos como sendo:

$$
Q_{y}=\left[\begin{array}{cc}
7 & 0 \\
0 & 0,4
\end{array}\right] \quad Q_{r}=\left[\begin{array}{ll}
1 & 0 \\
0 & 1
\end{array}\right] \quad Q_{x}=\left[\begin{array}{lll}
1 & 0 & 0 \\
0 & 1 & 0 \\
0 & 0 & 1
\end{array}\right]
$$

onde $Q_{y}$ é o peso nas saídas, $Q_{r}$ o peso nas entradas e $Q_{x}$ o peso nos estados.

O controlador preditivo e as simulações foram desenvolvidas usando um toolbox para MatLab®, o MPT Toolbox, desenvolvido por [12].

As simulações foram realizadas com um tempo de amostragem de $0.5 \mathrm{~s}$. O set point da posição foi dado na forma de degrau com a coordenada $(2,1)$.

A Figura 3 mostra a simulação do sistema que apresentou um offset total menor que 5\%, o tempo de cálculo off-line do controlador foi de 5,4min e o tempo para fazer os cálculos online foi de $56 \mathrm{~ms}$ por amostragem.
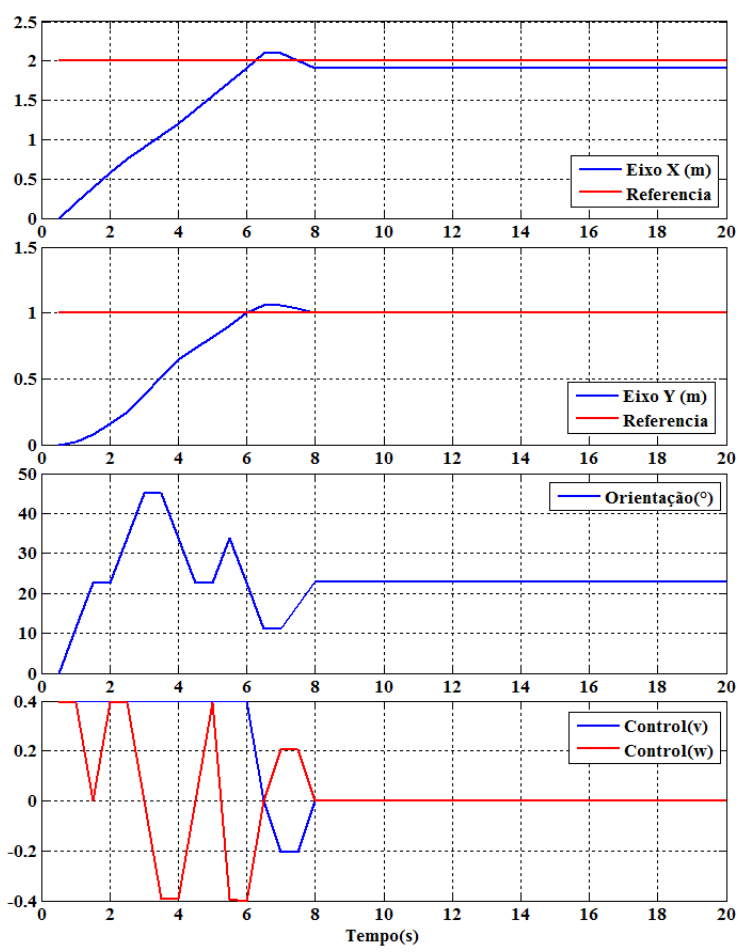

Figura 3. Resposta ao degrau de $(2,1)$.

Uma nova simulação foi realizada, agora para um degrau na coordenada $(1,3)$ e observa-se que o sistema estabilizou com um grande erro de offset, sendo necessária uma nova sintonia, Figura 4, este fato ocorreu todas as vezes que houve uma mudança no offset.

Para o controlador desenvolvido, os estados foram separados em 94.629 regiões, cada uma com sua ação de controle (10), Figura 5. 


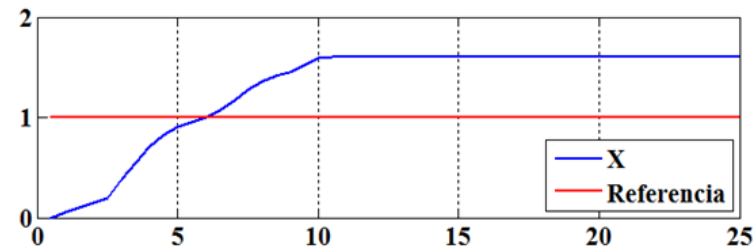

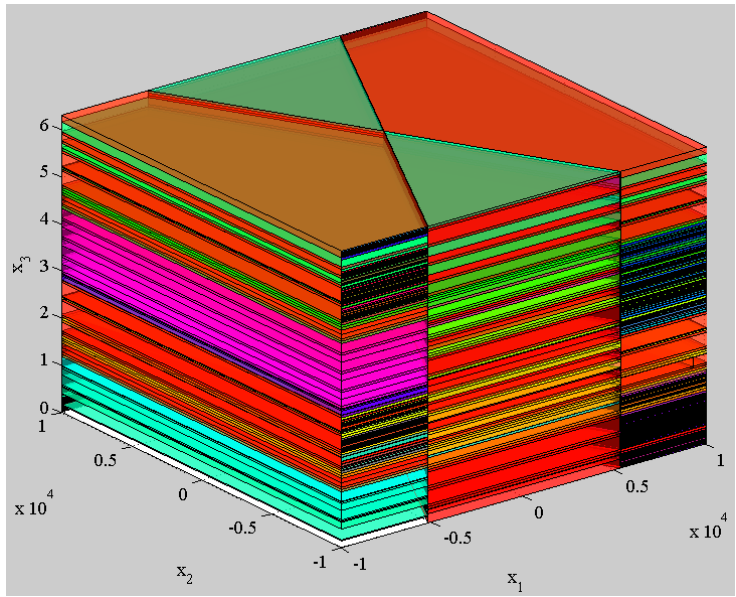

Figura 5. Regiões do espaço de estados.
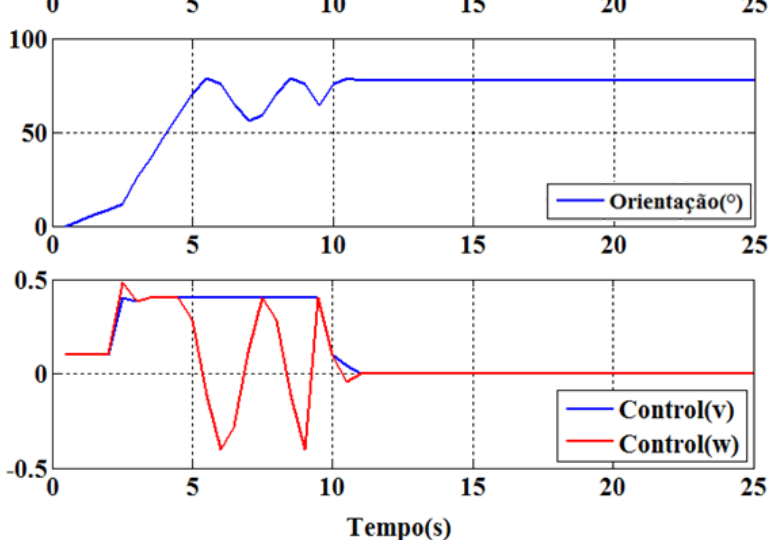

Figura 4. Resposta ao degrau de (1,3).

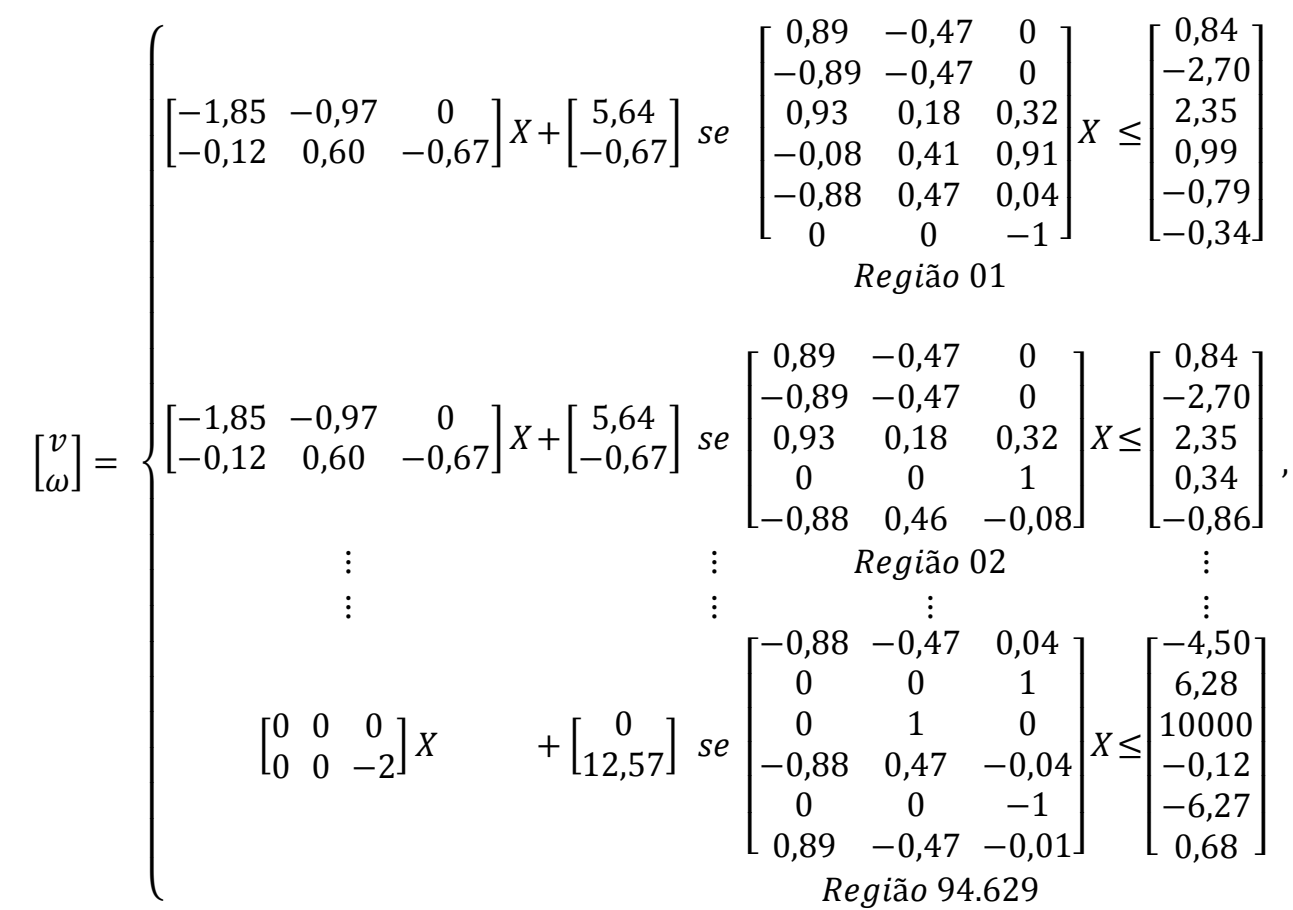

onde $X=\left[\begin{array}{lll}x & y & \varphi\end{array}\right]^{T}$.

\section{Conclusão}

Este artigo demostrou como projetar um controlador preditivo para um sistema restrito com múltiplas entradas e múltiplas saídas.
Percebe-se através da simulação, que o algoritmo do controlador preditivo consegue realizar o controle do sistema cinemático de uma cadeira de rodas robótica de forma estável e respeitando as restrições.

O controlador gerado com o algoritmo de otimização por Programação Multiparamétrica agregou agilidade e eficiência para o sistema, uma vez que as ações de controle são previamente conhecidas. 
É importante ressaltar que os resultados foram obtidos através de parâmetros estipulados por tentativa e erro, sendo assim, o sistema poderia ter a mesma eficiência, ou melhor, utilizando menos tempo computacional se alguma ferramenta de identificação estipulasse os parâmetros ótimos, como algoritmo genético, por exemplo.

Contudo, quando foram feitos testes de mudança de coordenadas (setpoint), observou-se um grande erro de offset, tendo de se realizar nova sintonia. Tal fato ocorreu sempre que foi feita uma mudança drástica na entrada.

Isso se mostrou um problema grave, podendo ser tópico de trabalhos futuros.

\section{Referências Bibliográficas}

Akiba, S., Zanma, T. \& Ishida, M., 2010. Model Predictive Control Based Optimal Crusing Control Of Two-Wheeled Mobile Robots. Pp. 170-175.

Bemporad, A., Morari, M., Dua, V. \& Pistikopoulos, E., 2002. The Explicit Linear Quadratic Regulator For Contrained Systems.. Automática, Janeiro, Volume 38(1), Pp. 3-20. DOI: 10.1016/S0005-1098(01)00174-1

Borrelli, F., 2003. Constrained Optimal Control Of Linear And Hybrid Systems. $1^{\text {a }}$ Ed. S.L.:Springer.

Camacho, E. F. \& Bordons, C., 2004. Model Predictive Control. $2^{\mathrm{a}}$ Ed. S.L.:Springer.

Celeste, W. C., 2009. Um Sistema Autônomo Para Navegação De Cadeiras De Rodas Robóticas Orientadas A Pessoas Com Deficiência Motora Severa, S.L.: S.N.

Chen, X. \& Li, Y., 2007. Neural Network Predictive Control For Mobile Robot Using Pso With Controllable Random Exploration Velocity. International Journaol Of Intelligent Control And Systems, Set., 12(3), Pp. 217-229.

Chiou, J.-S. \& Wang, K.-Y., 2008. Application Of A Hybrid Controller To A Mobile Robot.
Simulation Modelling Practice And Theory, Volume 16, Pp. 783-795. DOI: 10.1016/j.simpat.2008.04.018

Kanjanawanishkul, K. \& Zell, A., 2008. Distributed

Model Predictive Control For Coordinated Path Following Control Of Omnidirectional Mobile Robots. Ieee International Conference On Systems, Man And Cybernetics, Out.Pp. 3120-

Klancar, G. \& Skrjanc, I., 2007. Tracking-Error Model-Based Predictive Control For Mobile Robots In Real Time. Robotics And Autonomous Systems 55, Pp. 460-469. DOI: 10.1016/j.robot.2007.01.002

Kühne, F., Da Silva, J. M. G. \& Lages, W. F., 2005. Mobile Robot Trajectory Tracking Using Model Predictive Control. Ieee Latin-American Robotics Symposium, Set.

Kvasnica, M., 2009. Real-Time Model Predictive Control Via Multi-Parametric Programming. $1^{\text {a }}$ Ed. Saarbrücken, Alemanha: Vdm.

Martins, F. Et Al., 2008. An Adaptive Dynamic Controller For Autonomous Mobile Robot Trajectory Tracking. Control Engineering Practice, Volume 16, Pp. 1354-1363. DOI: 10.1016/j.conengprac.2008.03.004

Nourbakhsh, I. R. \& Siegwart, R., 2004. Introduction To Autonomous Mobile Robots. Cambridge: The Mit Press.

Rico, J. E. N., Ortega, J. G. \& Camacho, E. F., 1999. A Smith-Predictor-Based Generalized Predictive Controller For Mobile Robot Path-Tracking. Control Engineering Practice 7, Pp. 729-740. DOI: 10.1016/S0967-0661(99)00025-8

Siciliano, B. \& Khatib, O., 2008. Springer Handbook Of Robotics. Berlin: Springer. DOI: 10.1007/978-3-540-30301-5

Yoo, S. J., Choi, Y. H. \& Park, J. B., 2006. Generalized Predictive Control Based On SelfRecurrent Wavelet Neural Network For Stable Path Tracking Of Mobile Robots: Adaptive Learning Rates Approach. Ieee Transactions On Circuits And Systems, Jun, 53(6), Pp. 1381-1394. DOI: 10.1109/TCSI.2006.875166 